\title{
Article \\ Gold(I)-Catalyzed Tandem Synthesis of Polycyclic Dihydroquinazolinones
}

\author{
Jingyang Sun, Yoona Song and Jae-Sang Ryu *(D) \\ College of Pharmacy \& Graduate School of Pharmaceutical Sciences, Ewha Womans University, \\ 52 Ewhayeodae-gil, Seodaemun-Gu, Seoul 03760, Korea; sunjingyangs@163.com (J.S.); syn3829@naver.com (Y.S.) \\ * Correspondence: ryuj@ewha.ac.kr; Tel.: +82-2-3277-3008
}

Citation: Sun, J.; Song, Y.; Ryu, J.-S Gold(I)-Catalyzed Tandem Synthesis of Polycyclic Dihydroquinazolinones. Catalysts 2021, 11, 1436. https:// doi.org/10.3390/catal11121436

Academic Editor: Luca Gonsalvi

Received: 22 October 2021

Accepted: 23 November 2021

Published: 25 November 2021

Publisher's Note: MDPI stays neutral with regard to jurisdictional claims in published maps and institutional affiliations.

Copyright: (c) 2021 by the authors Licensee MDPI, Basel, Switzerland. This article is an open access article distributed under the terms and conditions of the Creative Commons Attribution (CC BY) license (https:/ / creativecommons.org/licenses/by/ $4.0 /)$.
Abstract: A gold-catalyzed cascade process for the synthesis of dihydroquinazolinone scaffolds was developed. A series of gold catalysts were screened for this tandem transformation, and the $\left(\mathrm{PPh}_{3}\right) \mathrm{AuCl} / \mathrm{AgOTf}$ catalyst combination was found to be the best catalyst system. This method is characterized by good yields, high regioselectivity, and broad substrate scope. This method is also applicable to the synthesis of tetracyclic dihydroquinazolinones and seven-membered ring-fused dihydroquinazolinones.

Keywords: gold; silver; dihydroquinazolinone; tandem reaction; cascade reaction

\section{Introduction}

Nitrogen-containing heterocycles [1-3] are important molecular architectures frequently found in bioactive natural products and drug candidates. As bioactive alkaloids [4-6], dihydroquinazolinones [7-9] are not only an important class of compounds but also substructures of structurally complex polycyclic alkaloids with a wide range of biological activities. More specifically, fused dihydroquinazolinones such as cruciferane [10], phaitanthrin D [11], evodiamine [12], and their synthetic congeners [13,14] have attracted medicinal chemists' attention due to their unique structural features and promising bioactivities, which have prompted the research on synthetic dihydroquinazolinones with pharmacological potential (Figure 1). Although various synthetic approaches to bicyclic dihydroquinazolinone scaffolds have been described [15-20], effective strategies for more complex tri- and polycyclic dihydroquinazolinones, which operate under mild conditions on readily accessible simple substrates, are rare and of high value.

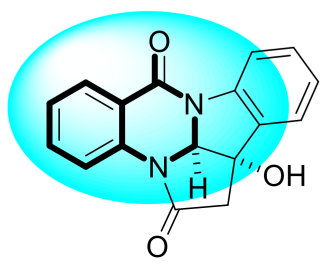

$( \pm)$-Cruciferane

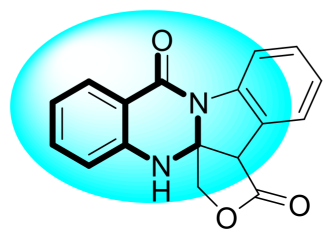

(-)-Phaitanthrin D

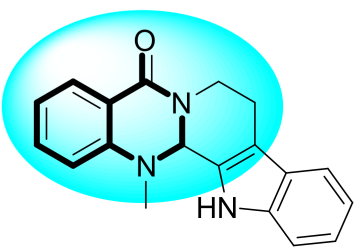

Evodiamine
Figure 1. Bioactive fused dihydroquinazolinone alkaloids.

Thus far, a number of synthetic methodologies for various heterocyclic systems have been developed. Nevertheless, highly selective and efficient synthetic strategies, which allow a rapid increase in molecular complexity with a reduced number of transformation steps and purification processes, are still in demand. In this context, clean 'one-pot cascade reactions' [21-25] can reduce the number of synthetic steps and tedious purification processes. These reactions rapidly increase molecular complexity and diversity in a single operation in which reactive intermediates are produced and utilized for the next step of 
reaction without isolation. 'One-pot cascade reactions' may also save the time and labor required for multistep synthesis and minimize waste production during synthesis. Due to these advantages, 'one-pot cascade reactions' have emerged as an important tool for the efficient construction of more complicated polycyclic molecular skeletons.

Over the past two decades, homogeneous gold catalysis [26,27] has become one of the most promising fields in organic and organometallic chemistry. Due to its high reactivity toward $\pi$-systems and broad functional group compatibility, homogeneous gold catalysis has been established as a powerful tool for one-pot cascade reactions [28,29]. Gold catalysts act as carbophilic $\pi$-Lewis acids and efficiently activate $C-C$ multiple bonds to form reactive intermediates [30], which further promote subsequent reactions with various types of partners. Specifically, when the activated C-C multiple bonds are combined with heteronucleophiles, enol/enamine-type reactive species are generated in situ, and several types of cascade cyclization reactions can be promoted (Scheme 1) [31,32]. Therefore, enol/enaminetype reactive species are considered as synthetically valuable and useful for gold-catalyzed one-pot cascade reactions $[33,34]$. Here, we have attempted to develop an efficient one-pot cascade synthetic method and report a gold-catalyzed one-pot cascade process for the synthesis of dihydroquinazolinones, which involves a double hydroamination process.

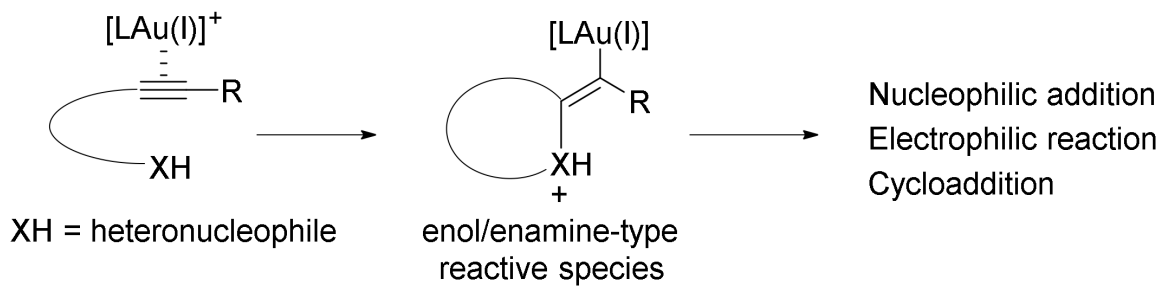

Scheme 1. Gold-catalyzed one-pot cascade reaction using enol/enamine-type species.

\section{Results and Discussion}

To investigate the feasibility of the gold-catalyzed one-pot cascade process for the synthesis of dihydroquinazolinone scaffolds, we initially focused on the double cascade cyclization of alkyne-tethered anthranilamide 1a as a benchmark substrate, which was readily prepared in one step from commercially available isatoic anhydride and 1-amino-4pentyne. When 1a was subjected to a catalyst mixture of $\left(\mathrm{PPh}_{3}\right) \mathrm{AuCl} / \mathrm{AgOTf}(10 \mathrm{~mol} \%)$ dissolved in toluene, the substrate $1 \mathrm{a}$ was completely consumed at $60{ }^{\circ} \mathrm{C}$ in $5 \mathrm{~h}$. The desired dihydroquinazolinone product $2 \mathrm{a}$ was obtained in $52 \%$ yield along with $6 \%$ of ketone byproduct 3a (Table 1, entry 1), which presumably was formed from the goldcatalyzed addition of $\mathrm{H}_{2} \mathrm{O}$ to the alkyne moiety of 1a. Although the formation of the ketone 3a was suppressed by the addition of the $4 \AA$ molecular sieve, it could not be completely prevented.

Initially, we optimized solvent conditions to screen for various cationic gold catalysts and silver cocatalysts. The double cascade cyclization reaction of $\mathbf{1 a}$ was investigated in various solvents including $\mathrm{CH}_{3} \mathrm{CN}$, THF, $\mathrm{CH}_{2} \mathrm{Cl}_{2}, 1$,4-dioxane, cyclopentyl methyl ether (CPME), dimethyl carbonate (DMC), and dichloroethane (DCE) (Table 1, entries 2-8). The reactions were effective in all the solvents except $\mathrm{CH}_{3} \mathrm{CN}$; only a small amount of product 2a was observed and isolated in the reaction in $\mathrm{CH}_{3} \mathrm{CN}$ (Table 1, entry 2). Considering the yield of the product, DCE was the best solvent for the reaction (Table 1, entry 8); thus, DCE was chosen for further study. We also briefly examined other Au/Ag catalyst combinations. The $\left(\mathrm{PPh}_{3}\right) \mathrm{AuCl}$ catalyst systems combined with $\mathrm{AgNO}_{3}, \mathrm{AgBF}_{4}, \mathrm{AgOTs}$, and $\mathrm{AgSbF}_{6}$ were examined for cyclization at $60^{\circ} \mathrm{C}$ in DCE solvent (Table 1, entries 9-12). The reactions using $\mathrm{AgNO}_{3}, \mathrm{AgBF}_{4}$, and $\mathrm{AgOTs}$ were fairly clean and comparable to the reaction with $\left(\mathrm{PPh}_{3}\right) \mathrm{AuCl} / \mathrm{AgOTf}$. Particularly, the reaction with $\left(\mathrm{PPh}_{3}\right) \mathrm{AuCl} / \mathrm{AgNO} \mathrm{N}_{3}$ proceeded smoothly but slowly and provided the cyclic dihydroquinazolinone product 2a in $80 \%$ yield after $10 \mathrm{~h}$ (Table 1 , entry 9 ). 
Table 1. Optimization of reaction conditions ${ }^{1}$.

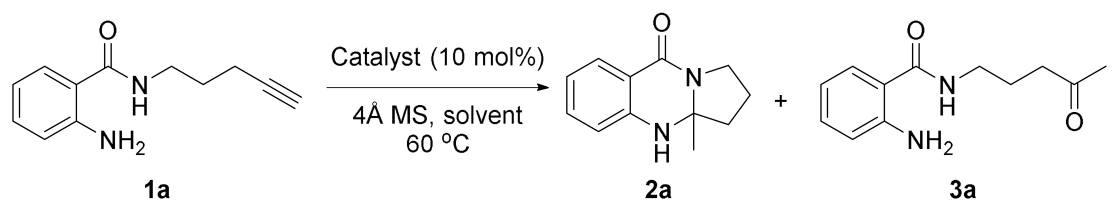

\begin{tabular}{|c|c|c|c|c|}
\hline \multirow{2}{*}{ Entry } & \multirow{2}{*}{ Catalyst } & \multirow{2}{*}{ Time (h) } & \multicolumn{2}{|c|}{ Yield (\%) ${ }^{2}$} \\
\hline & & & $2 a$ & $3 a$ \\
\hline 1 & $\left(\mathrm{PPh}_{3}\right) \mathrm{AuCl} / \mathrm{AgOTftoluene}$ & 5 & 52 & 6 \\
\hline 2 & $\left(\mathrm{PPh}_{3}\right) \mathrm{AuCl} / \mathrm{AgOTCH}{ }_{3} \mathrm{CN}$ & 24 & 15 & 10 \\
\hline 3 & $\left(\mathrm{PPh}_{3}\right) \mathrm{AuCl} / \mathrm{AgOTf} \mathrm{THF}$ & 2 & 67 & 5 \\
\hline 4 & $\left(\mathrm{PPh}_{3}\right) \mathrm{AuCl} / \mathrm{AgOTK}_{2} \mathrm{Cl}_{2}$ & 4 & 68 & 2 \\
\hline 5 & $\left(\mathrm{PPh}_{3}\right) \mathrm{AuCl} / \mathrm{AgOTflioxane}$ & 1 & 76 & 8 \\
\hline 6 & $\left(\mathrm{PPh}_{3}\right) \mathrm{AuCl} / \mathrm{AgOTfCPME}$ & 18 & 33 & 4 \\
\hline 7 & $\left(\mathrm{PPh}_{3}\right) \mathrm{AuCl} / \mathrm{AgOTf} \mathrm{DMC}$ & 4 & 66 & 0 \\
\hline 8 & $\left(\mathrm{PPh}_{3}\right) \mathrm{AuCl} / \mathrm{AgOTf} \mathrm{DCE}$ & 1 & 80 & 0 \\
\hline 9 & $\left(\mathrm{PPh}_{3}\right) \mathrm{AuCl} / \mathrm{AgNO}_{3} \mathrm{DCE}$ & 10 & 80 & 1 \\
\hline 10 & $\left(\mathrm{PPh}_{3}\right) \mathrm{AuCl} / \mathrm{AgBF}_{4} \mathrm{DCE}$ & 1 & 76 & 2 \\
\hline 11 & $\left(\mathrm{PPh}_{3}\right) \mathrm{AuCl} / \mathrm{AgOTs} \mathrm{DCE}$ & 1 & 74 & 1 \\
\hline 12 & $\left(\mathrm{PPh}_{3}\right) \mathrm{AuCl} / \mathrm{AgSbF}_{6} \mathrm{DCE}$ & 24 & 40 & 15 \\
\hline 13 & $\left(\mathrm{PPh}_{3}\right) \mathrm{AuCl} \quad \mathrm{DCE}$ & 24 & 17 & 1 \\
\hline 14 & AgOTf & 24 & 23 & 0 \\
\hline 15 & $\left(\mathrm{PPh}_{3}\right) \mathrm{AuNTf}_{2}$ & 2 & 78 & 7 \\
\hline 16 & (XPhos)AuCl/AgOTACE & 1 & 56 & 7 \\
\hline 17 & (IPr)AuCl/AgOTf DCE & 3 & 80 & 4 \\
\hline 18 & (IPr)AuCl/AgNTf 2 DCE & 1 & 68 & 4 \\
\hline 19 & (IPr)AuCl/ $\mathrm{AgNO}_{3} \quad \mathrm{DCE}$ & 24 & 20 & 4 \\
\hline $20^{3}$ & TfOH DCE & 24 & 4 & 0 \\
\hline $21^{4}$ & $\left(\mathrm{PPh}_{3}\right) \mathrm{AuCl} / \mathrm{AgOTf} \mathrm{DCE}$ & 1 & 76 & 0 \\
\hline $22^{5}$ & $\left(\mathrm{PPh}_{3}\right) \mathrm{AuCl} / \mathrm{AgOTf} \mathrm{DCE}$ & 1 & 67 & 6 \\
\hline
\end{tabular}

${ }^{1}$ Reaction conditions: 1a $(80.9 \mathrm{mg}, 400 \mu \mathrm{mol})$, Au catalyst $(40.0 \mu \mathrm{mol}), \mathrm{Ag}$ catalyst $(40.0 \mu \mathrm{mol})$, solvent $(4 \mathrm{~mL}), 4 \AA$ molecular sieve $(500 \mathrm{mg}) .{ }^{2}$ Isolated yields. ${ }^{3}$ Starting material was recovered. ${ }^{4}$ A total of 5 mol $\%$ of catalyst was loaded. ${ }^{5}$ A total of $1 \mathrm{~mol} \%$ of catalyst was loaded.

Although the reaction was not effective with $\left(\mathrm{PPh}_{3}\right) \mathrm{AuCl}$ or AgOTf alone, a single $\left(\mathrm{PPh}_{3}\right) \mathrm{AuNTf}_{2}$ catalyst was as effective as the $\left(\mathrm{PPh}_{3}\right) \mathrm{AuCl} / \mathrm{AgOTf}$ catalyst combination, which gave the desired double cyclized product $\mathbf{2 a}$ without silver (Table 1, entries 13 and 14 vs. entry 15). In comparison with $\left(\mathrm{PPh}_{3}\right) \mathrm{AuCl}$ or AgOTf alone, the improved reactivity of the $\left(\mathrm{PPh}_{3}\right) \mathrm{AuCl} / \mathrm{AgOTf}$ catalyst combination may be attributed to the anion effect rather than the silver effect. Changing $\mathrm{PPh}_{3}$ with a biphenyl monodentate phosphine ligand (XPhos) did not improve the reactivity (Table 1, entry 16). The phosphine ligand was not essential for the reaction. Replacing the phosphine ligand $\mathrm{PPh}_{3}$ with $\mathrm{IPr}$ did not change the reactivity significantly (Table 1, entries 17-19). Interestingly, $10 \mathrm{~mol} \%$ Brønsted acid $(\mathrm{TfOH})$ did not catalyze the cyclization efficiently and generated the cyclized product $\mathbf{2 a}$ only in a trace amount (Table 1, entry 20). In addition, the effect of the catalyst loading on catalytic activity was also examined using $5 \mathrm{~mol} \%$ and $1 \mathrm{~mol} \%$ of $\left(\mathrm{PPh}_{3}\right) \mathrm{AuCl} / \mathrm{AgOTf}$ (Table 1, entries 21 and 22). It is noteworthy that catalyst loading could be reduced to as low as $1 \mathrm{~mol} \%$ with a slight decrease in yield (67\%; Table 1, entry 22). Based on these observations, we chose to set the catalyst loading at $10 \mathrm{~mol} \%$ for sterically hindered and unreactive substrates as shown in Table 2. 
Table 2. Reaction scope ${ }^{1}$.

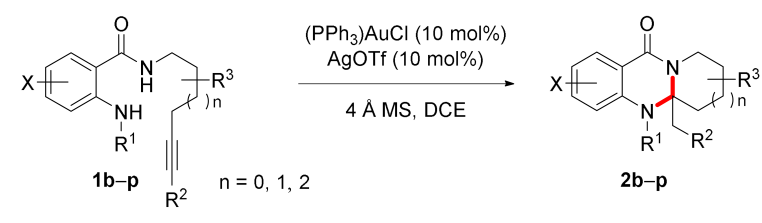

\begin{tabular}{|c|c|c|c|c|c|}
\hline Entry & Substrate & Product & $\mathrm{T}\left({ }^{\circ} \mathrm{C}\right)$ & Time (h) & Yield $(\%)^{2}$ \\
\hline 1 & & $2 b$ & $\mathrm{rt}$ & 1.5 & 85 \\
\hline 2 & 1c & $2 c$ & $\mathrm{rt}$ & 3 & 90 \\
\hline 3 & 1d & $2 d$ & $\mathrm{rt}$ & 1 & 98 \\
\hline 4 & $1 \mathrm{e}$ & $2 e$ & 60 & 24 & 80 \\
\hline $5^{3}$ & & & 120 & 4 & 10 \\
\hline $6^{4}$ & & $2 \mathrm{~g}$ & 120 & 1 & 30 \\
\hline 7 & 1h & $2 \mathrm{~h}$ & 120 & 2.5 & 94 \\
\hline 8 & $1 \mathrm{i}$ & $2 i$ & 120 & 1.25 & 84 \\
\hline 9 & $1 \mathrm{j}$ & $2 j$ & 120 & 0.3 & 57 \\
\hline 10 & $1 \mathrm{k}$ & $2 k$ & 120 & 1 & 61 \\
\hline
\end{tabular}


Table 2. Cont.

\begin{tabular}{|c|c|c|c|c|c|}
\hline Entry & Substrate & Product & $\mathrm{T}\left({ }^{\circ} \mathrm{C}\right)$ & Time (h) & Yield $(\%)^{2}$ \\
\hline $11^{5}$ & 11 & 21 & 120 & 1 & 31 \\
\hline 12 & $1 \mathrm{~m}$ & $2 m$ & rt & 0.5 & 96 \\
\hline 13 & $1 \mathrm{n}$ & $2 n$ & rt & 0.5 & 97 \\
\hline 14 & 10 & 20 & $\mathrm{rt}$ & 2 & 92 \\
\hline 15 & $1 p$ & $2 p$ & $\mathrm{rt}$ & 0.5 & 97 \\
\hline 16 & $1 q$ & $2 q$ & $\mathrm{rt}$ & 8 & 91 \\
\hline 17 & $1 \mathrm{r}$ & $2 r$ & rt & 24 & 76 \\
\hline 18 & is & $2 s$ & $\mathrm{rt}$ & 3 & 72 \\
\hline 19 & Me $1 \mathrm{t}$ & $2 t$ & $\mathrm{rt}$ & 1 & 86 \\
\hline 20 & 14 & 24 & 60 & 1.5 & 97 \\
\hline
\end{tabular}

${ }^{1}$ Reaction conditions: 1b-u $(400 \mu \mathrm{mol}),\left(\mathrm{PPh}_{3}\right) \mathrm{AuCl}(19.8 \mathrm{mg}, 40.0 \mu \mathrm{mol}, 10 \mathrm{~mol} \%), \mathrm{Ag}$ catalyst $(10.3 \mathrm{mg}$, $40.0 \mu \mathrm{mol}, 10 \mathrm{~mol} \%), \mathrm{DCE}(4 \mathrm{~mL}), 4 \AA$ molecular sieve $(500 \mathrm{mg}) .{ }^{2}$ Isolated yields. ${ }^{3} 6 \%$ of ketone $3 \mathrm{f}$ was also isolated. ${ }^{4} 15 \%$ of ketone $3 \mathrm{~g}$ was also isolated. ${ }^{5} 17 \%$ of ketone 31 was also isolated. 
After identifying $\left(\mathrm{PPh}_{3}\right) \mathrm{AuCl} / \mathrm{AgOTf}$ as the best catalyst, we investigated the scope of gold(I)-catalyzed one-pot cascade reactions for alkyne-tethered anthranilamides using $\left(\mathrm{PPh}_{3}\right) \mathrm{AuCl} / \mathrm{AgOTf}$ at room temperature under optimized conditions (Table 2). The double cascade cyclization reaction worked well with a wide variety of alkyne-tethered anthranilamide substrates and showed broad functional group compatibility. When the $\left(\mathrm{PPh}_{3}\right) \mathrm{AuCl} / \mathrm{AgOTf}$ catalyst combination was used at room temperature, the substrates $\mathbf{1 b}$ and $\mathbf{1 c}$ containing pentyne tethers smoothly provided double cyclized products (fivemembered ring-fused dihydroquinazolinones $\mathbf{2 b}$ and $\mathbf{2 c}$ ) in excellent yields ( $85 \%$ and $90 \%$, entries 1 and 2). Likewise, the substrate $1 \mathbf{d}$ containing a fused-aromatic substituent on the tether also readily underwent double cascade cyclization and afforded the corresponding dihydroquinazolinone product $\mathbf{2 d}$ in $98 \%$ yield (entry 3 ). Furthermore, the double cascade cyclization reaction worked well with the internal alkyne substrate 1e, which was smoothly cyclized to $2 \mathbf{e}$ at $60{ }^{\circ} \mathrm{C}$ in $80 \%$ yield (entry 4 ). However, the reaction with the phenyl substituted internal alkyne substrate $\mathbf{1 f}$ needed a higher temperature for cyclization due to the unfavorable electronic and steric effects of the phenyl group. Interestingly, the reaction of $\mathbf{1 f}$ provided the five-membered ring-fused dihydroquinazolinone $\mathbf{2 f}$ (entry 5). Although coordination of the $\pi$-acidic cationic gold catalyst to alkyne may increase the electrophilic nature of the benzylic position, 5-exo-dig cyclization was more favored than 6-endo-dig cyclization (Scheme 2).

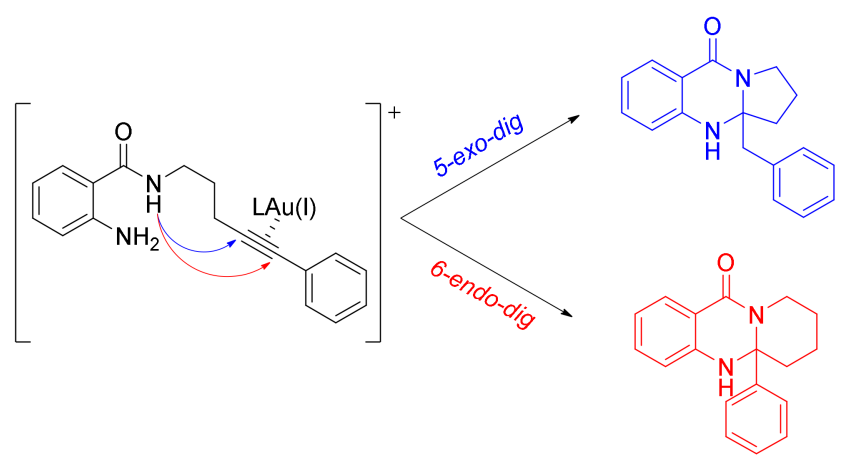

Scheme 2. Regioselectivity in ring closure.

On the other hand, the double cascade reaction was also effective for the synthesis of six-membered ring-fused dihydroquinazolinones. However, six-membered ring formation was slower than five-membered ring formation at ambient temperature owing to the higher energy barrier for ring closure. Therefore, the reaction was carried out at $120^{\circ} \mathrm{C}$ under sealed-tube conditions in a microwave reactor; the substrates $\mathbf{1 g}-\mathbf{j}$ containing hexyne tethers and $1 \mathbf{k}$ containing a fused-aromatic substituent on the tether were converted into the corresponding six-membered ring-fused dihydroquinazolinones $\mathbf{2 g}-\mathbf{k}$ in moderate yields (30-75\%, entries 6-10). Notably, this reaction worked well for seven-membered ring formation as well. Despite unfavorable enthalpic and entropic factors associated with seven-membered ring closure [35-37], our method was applicable to the synthesis of the seven-membered ring-fused dihydroquinazolinone 21 , which was obtained in $31 \%$ yield in the presence of (IPr) AuCl/AgOTf at $120^{\circ} \mathrm{C}$ in $1 \mathrm{~h}$ (entry 11).

Next, we investigated the substrate scope with respect to the electronic effect of substituents. The method showed good compatibility for electron-withdrawing and electrondonating functional groups such as chloro, bromo, nitro, alkyl, and methoxy groups. The substrates containing electron-withdrawing substituents $(\mathbf{1} \mathbf{m}-\mathbf{p})$ smoothly afforded the cyclized products $\mathbf{2} \mathbf{m}-\mathbf{p}$ at room temperature in excellent yields (92-97\%, entries $12-15)$. The substrates containing electron-donating substituents $(\mathbf{1} \mathbf{q}-\mathbf{t})$ also provided the corresponding cyclized products in good to excellent yields (72-91\%, entries 16-19). Furthermore, the reaction was tolerant of the $\mathrm{N}$-alkyl substituent of the anthranilamide substrate; the $\mathrm{N}$ methyl substituent of the anthranilamide substrate $1 \mathbf{u}$ did not affect the second cyclization reaction and afforded the dihydroquinazolinone $2 \mathbf{u}$ in $97 \%$ yield at $60^{\circ} \mathrm{C}$. 
Based on our observation and previous reports about gold(I)-catalyzed alkyne hydroamination [38,39], the possible mechanism is proposed in Scheme 3. First, the gold(I) chloride complex precursor condenses with silver salts, which would scavenge chloride ions as insoluble $\mathrm{AgCl}$ and generate the active gold(I) catalyst $\mathbf{A}$. The coordination of the active gold(I) species $\mathbf{A}$ to the alkyne moiety of the substrate 1a leads to the formation of the gold $\pi$-alkyne complex $\mathbf{B}$, which could be cyclized to the gold-alkyl complex $\mathbf{C}$ or hydrated to the ketone $3 \mathbf{a}$ by the adventitious addition of water. Upon the protodeauration of $\mathbf{C}$, the enamine intermediate $\mathbf{D}$ is released, and the active gold(I) catalyst $\mathbf{A}$ is regenerated. Next, the second cyclization reaction would begin with the re-coordination of the $\pi$-acidic cationic gold catalyst $\mathbf{A}$ to the alkene moiety of the enamine intermediate $\mathbf{D}$, which catalyzes the formation of the iminium intermediate $\mathbf{E}$ and accelerates the second intramolecular cyclization reaction. The resultant gold-alkyl complex $\mathbf{F}$ readily undergoes protodeauration and decomposes to yield the double cyclized product $\mathbf{2} \mathbf{a}$ and the cationic gold(I) catalyst A. This proposed mechanism involves repeated coordination and subsequent cyclization mediated by the gold(I) catalyst.

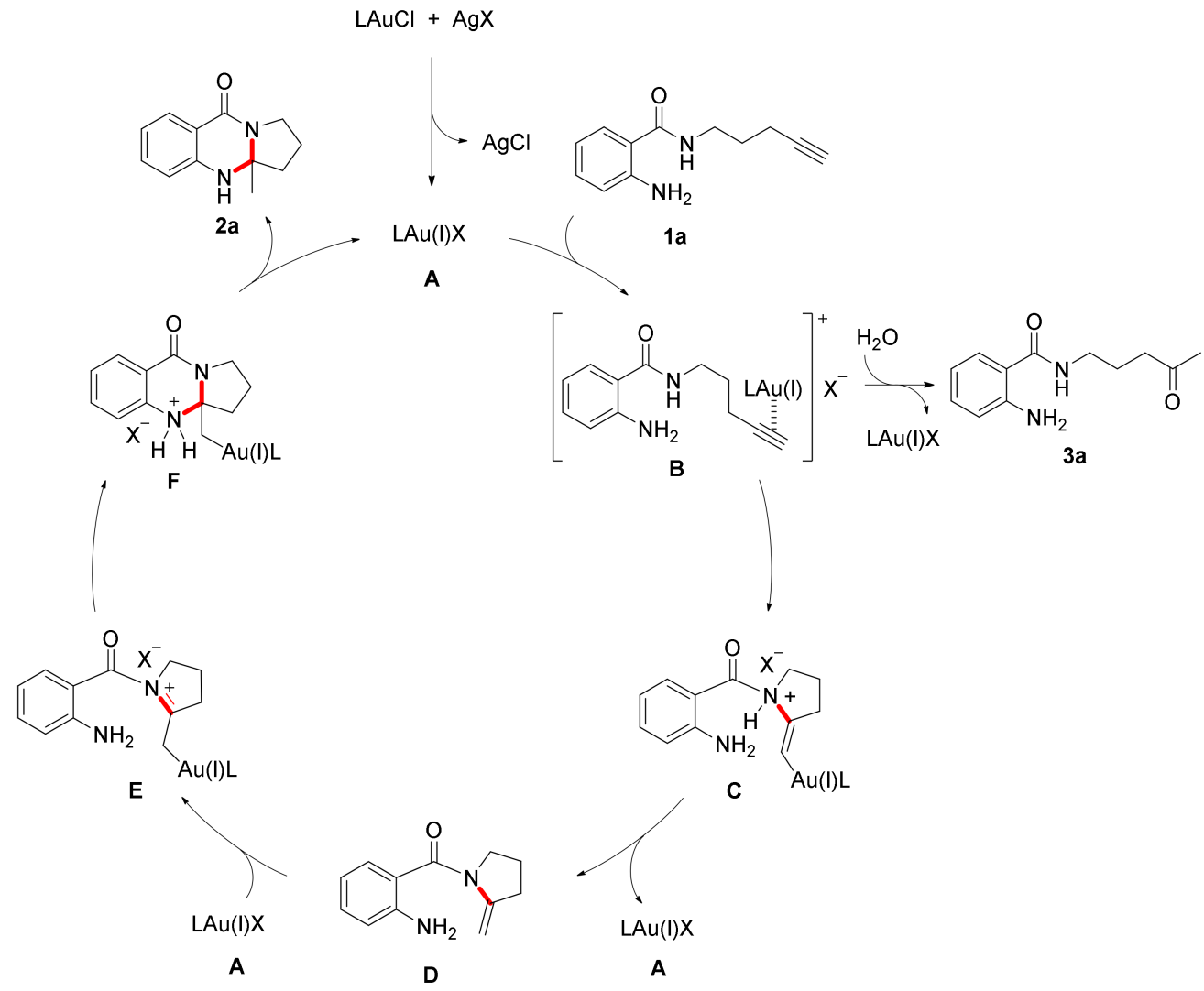

Scheme 3. Proposed mechanism.

\section{Materials and Methods}

\subsection{General Information}

All reactions were performed in oven-dried glassware fitted with glass stoppers under positive pressure of Ar with magnetic stirring, unless otherwise noted. Air- and moisture-sensitive liquids and solutions were transferred via syringe or stainless-steel cannula. TLC was performed on $0.25 \mathrm{~mm}$ E. Merck (Darmstadt, Germany) silica gel 60 $\mathrm{F}_{254}$ plates and visualized under UV light $(254 \mathrm{~nm})$ or by staining with cerium ammonium molybdenate (CAM), potassium permanganate $\left(\mathrm{KMnO}_{4}\right)$, ninhydrin, or $p$-anisaldehyde. Flash chromatography was performed on E. Merck (Darmstadt, Germany) 230-400 mesh silica gel 60. Medium-pressure liquid chromatography (MPLC) was performed on a prepacked column (silica gel, $10 \mu \mathrm{m}$ ) with a UV detector. Reagents were purchased 
from commercial suppliers and used without further purification unless otherwise noted. Solvents were distilled from proper drying agents $\left(\mathrm{CaH}_{2}\right.$ or Na wire) under Ar atmosphere at $760 \mathrm{mmHg}$. All moisture- and/or oxygen-sensitive solids were handled and stored in a glovebox under $\mathrm{N}_{2}$. NMR spectra were recorded on Agilent (Santa Clara, CA, USA) Unity 400 instruments or Bruker (Billerica, MA, USA) Avene II $400 \mathrm{MHz}$ NMR spectrometer system equipped at Ewha Drug Development Research Core Center at $24^{\circ} \mathrm{C}$. Chemical shifts are expressed in ppm relative to TMS $\left({ }^{1} \mathrm{H}, 0 \mathrm{ppm}\right), \mathrm{CDCl}_{3}\left({ }^{1} \mathrm{H}, 7.26 \mathrm{ppm} ;{ }^{13} \mathrm{C}\right.$, $77.2 \mathrm{ppm})$, DMSO- $d_{6}\left({ }^{1} \mathrm{H}, 2.50 \mathrm{ppm} ;{ }^{13} \mathrm{C}, 39.5 \mathrm{ppm}\right), \mathrm{C}_{6} \mathrm{D}_{6}\left({ }^{1} \mathrm{H}, 7.16 \mathrm{ppm} ;{ }^{13} \mathrm{C}, 128.1 \mathrm{ppm}\right)$, $\mathrm{CD}_{3} \mathrm{OD}\left({ }^{1} \mathrm{H}, 3.31 \mathrm{ppm} ;{ }^{13} \mathrm{C}, 49.1 \mathrm{ppm}\right)$; coupling constants are expressed in Hz. High resolution mass spectra (HRMS) were obtained by electrospray ionization (ESI, TOF), electron ionization (EI, magnetic sector), Chemical ionization (CI, magnetic sector), or fast atom bombardment (FAB, magnetic sector). Infrared spectra were recorded with peaks reported in $\mathrm{cm}^{-1}$ (see Supplementary Materials).

\subsection{Representative Procedure for the Synthesis of Dihydroquinazolinone $\mathbf{2} \boldsymbol{a}-\boldsymbol{u}$}

To a $10 \mathrm{~mL}$ oven-dried round-bottom flask with a side arm were added $\mathbf{1 b}(92.1 \mathrm{mg}$, $400 \mu \mathrm{mol})$ and $4 \AA \mathrm{MS}(500 \mathrm{mg})$. The flask was brought into the glovebox, and $\left(\mathrm{PPh}_{3}\right) \mathrm{AuCl}$ (19.8 mg, $40.0 \mu \mathrm{mol}, 10 \mathrm{~mol} \%)$ and AgOTf (10.3 mg, $40.0 \mu \mathrm{mol}, 10 \mathrm{~mol} \%)$ were added inside a glovebox. Then, the flask was brought out of the glovebox, and anhydrous DCE $(4.0 \mathrm{~mL})$ was added. The reaction mixture was stirred at room temperature for $1.5 \mathrm{~h}$. Upon completion of the reaction, the precipitate was filtered off through a pad of Celite ${ }^{\circledR}$ and rinsed with $\mathrm{CH}_{2} \mathrm{Cl}_{2}(50 \mathrm{~mL})$. The filtrate was concentrated by rotary evaporation. The residue was purified by column chromatography (1.5:1 hexanes/EtOAc) to afford dihydroquinazolinone $\mathbf{2 b}(78.5 \mathrm{mg}, 340 \mu \mathrm{mol}, 85 \%)$ as a white solid.

\subsubsection{3a-Methyl-2,3,3a,4-tetrahydropyrrolo[2,1-b]quinazolin-9(1H)-one (2a)}

Reaction time: $1 \mathrm{~h}\left(60^{\circ} \mathrm{C}\right)$. White solid $(64.7 \mathrm{mg}, 80 \%)$. TLC: $R_{f} 0.08$ (1:1 hexane/EtOAc). mp: 168.9-170.9 ${ }^{\circ} \mathrm{C} .{ }^{1} \mathrm{H}$ NMR $\left(400 \mathrm{MHz}, \mathrm{C}_{6} \mathrm{D}_{6}\right): \delta 8.36(\mathrm{dd}, J=7.6,1.6 \mathrm{~Hz}, 1 \mathrm{H}), 7.09-6.98$ $(\mathrm{m}, 1 \mathrm{H}), 6.71(\mathrm{td}, J=7.5,1.0 \mathrm{~Hz}, 1 \mathrm{H}), 6.31-6.24(\mathrm{~m}, 1 \mathrm{H}), 3.69(\mathrm{dt}, J=12.2,8.1 \mathrm{~Hz}, 1 \mathrm{H}), 3.47$ (brs, $1 \mathrm{H}), 3.45-3.37(\mathrm{~m}, 1 \mathrm{H}), 1.60-1.51(\mathrm{~m}, 1 \mathrm{H}), 1.37-1.30(\mathrm{~m}, 2 \mathrm{H}), 1.27-1.18(\mathrm{~m}, 1 \mathrm{H}), 0.88(\mathrm{~s}$, 3H). ${ }^{13} \mathrm{C}$ NMR $\left(100 \mathrm{MHz}, \mathrm{C}_{6} \mathrm{D}_{6}\right): \delta 160.8,146.2,132.8,128.9,119.4,118.1,115.0,75.0,44.4$, 40.6, 24.9, 21.0. HRMS (ESI) $\mathrm{m} / \mathrm{z}$ calcd for $\mathrm{C}_{12} \mathrm{H}_{15} \mathrm{~N}_{2} \mathrm{O}[\mathrm{M}+\mathrm{H}]^{+}$203.1179, found 203.1179 .

Ketone 3a: Colorless liquid. TLC: $R_{f} 0.14$ (1:1 hexane/EtOAc). ${ }^{1} \mathrm{H}$ NMR $(400 \mathrm{MHz}$, $\left.\mathrm{C}_{6} \mathrm{D}_{6}\right): \delta 7.24-7.17(\mathrm{~m}, 1 \mathrm{H}), 7.12(\mathrm{td}, J=7.6,1.2 \mathrm{~Hz}, 1 \mathrm{H}), 6.60(\mathrm{td}, J=7.6,1.2 \mathrm{~Hz}, 1 \mathrm{H})$, 6.40-6.33 (m, 1H), 5.98 (brs, 1H), 5.65 (brs, 2H), $3.25(\mathrm{q}, J=6.4 \mathrm{~Hz}, 2 \mathrm{H}), 1.94(\mathrm{t}, J=6.8 \mathrm{~Hz}$, $2 \mathrm{H}), 1.67(\mathrm{~s}, 3 \mathrm{H}), 1.60(\mathrm{p}, J=6.8 \mathrm{~Hz}, 2 \mathrm{H}) .{ }^{13} \mathrm{C}$ NMR $\left(100 \mathrm{MHz}, \mathrm{C}_{6} \mathrm{D}_{6}\right): \delta 207.3,169.5,149.9$, $132.2,127.6,117.3,116.1,116.1,40.8,39.4,29.4,23.6$. HRMS (ESI) $\mathrm{m} / \mathrm{z}$ calcd for $\mathrm{C}_{12} \mathrm{H}_{17} \mathrm{~N}_{2} \mathrm{O}_{2}$ $[\mathrm{M}+\mathrm{H}]^{+}$221.1285, found 221.1287.

\subsubsection{2,2,3a-Trimethyl-2,3,3a,4-tetrahydropyrrolo[2,1-b]quinazolin-9(1H)-one (2b)}

Reaction time: $1.5 \mathrm{~h}$ (room temperature). White solid (78.5 mg, 85\%). TLC: $R_{f} 0.25$ (1.5:1 hexanes/EtOAc). mp: $172.3-174.3^{\circ} \mathrm{C} .{ }^{1} \mathrm{H}$ NMR $\left(400 \mathrm{MHz}, \mathrm{CDCl}_{3}\right): \delta 7.89$ (ddt, $J=7.8$, $1.5,0.6 \mathrm{~Hz}, 1 \mathrm{H}), 7.27(\mathrm{ddd}, J=8.0,7.3,1.6 \mathrm{~Hz}, 1 \mathrm{H}), 6.85(\mathrm{ddd}, J=7.8,7.3,1.0 \mathrm{~Hz}, 1 \mathrm{H}), 6.62$ $(\mathrm{ddd}, J=8.0,1.0,0.5 \mathrm{~Hz}, 1 \mathrm{H}), 4.18(\mathrm{brs}, 1 \mathrm{H}), 3.95(\mathrm{dd}, J=11.6,1.6 \mathrm{~Hz}, 1 \mathrm{H}), 3.13(\mathrm{dd}, J=11.6$, $0.8 \mathrm{~Hz}, 1 \mathrm{H}), 2.05(\mathrm{dd}, J=13.1 \mathrm{~Hz}, 1.6 \mathrm{~Hz}, 1 \mathrm{H}), 1.97(\mathrm{~d}, J=13.1 \mathrm{~Hz}, 2 \mathrm{H}), 1.48(\mathrm{~s}, 3 \mathrm{H}), 1.21$ (s, 3H), 1.19 (s, 3H). ${ }^{13} \mathrm{C}$ NMR (100 MHz, $\left.\mathrm{CDCl}_{3}\right): \delta 161.2,145.7,133.2,128.6,119.4,116.7$, 115.1, 76.1, 57.6, 55.6, 37.5, 28.3, 27.4, 27.2. HRMS (ESI) m/z calcd for $\mathrm{C}_{14} \mathrm{H}_{19} \mathrm{~N}_{2} \mathrm{O}[\mathrm{M}+\mathrm{H}]^{+}$ 231.1492, found 231.1488.

3.2.3. 3a'-Methyl-3a' , $^{\prime}$-dihydro-1'H-spiro[cyclohexane-1,2'-pyrrolo[2,1-b]quinazolin]$9^{\prime}\left(3^{\prime} \mathrm{H}\right)$-one $(2 \mathrm{c})$

Reaction time: $3 \mathrm{~h}$ (room temperature). White solid (96.9 mg, 90\%). TLC: $R_{f} 0.25$ (3:1 hexanes/EtOAc). mp: $241.1-243.1{ }^{\circ} \mathrm{C} .{ }^{1} \mathrm{H}$ NMR $\left(400 \mathrm{MHz}, \mathrm{C}_{6} \mathrm{D}_{6}\right): \delta 8.39(\mathrm{dd}, J=7.5,1.6 \mathrm{~Hz}$, 
1H), $7.05(\mathrm{ddd}, J=8.0,7.5,1.6 \mathrm{~Hz}, 1 \mathrm{H}), 6.72(\mathrm{td}, J=7.5,1.1 \mathrm{~Hz}, 1 \mathrm{H}), 6.21(\mathrm{dd}, J=8.0,1.1 \mathrm{~Hz}$, $1 \mathrm{H}), 4.26(\mathrm{~d}, J=11.9 \mathrm{~Hz}, 1 \mathrm{H}), 3.28(\mathrm{brs}, 1 \mathrm{H}), 2.85(\mathrm{~d}, J=11.9 \mathrm{~Hz}, 1 \mathrm{H}), 1.59(\mathrm{~d}, J=13.0 \mathrm{~Hz}$, $1 \mathrm{H}), 1.30(\mathrm{~d}, J=13.0 \mathrm{~Hz}, 1 \mathrm{H}), 1.38-1.04(\mathrm{~m}, 10 \mathrm{H}), 1.00(\mathrm{~s}, 3 \mathrm{H}) .{ }^{13} \mathrm{C} \mathrm{NMR}\left(100 \mathrm{MHz}, \mathrm{C}_{6} \mathrm{D}_{6}\right): \delta$ $160.6,146.0,132.8,129.2,119.4,117.6,114.9,75.0,40.7,37.3,36.3,26.8,26.1,24.1$, 23.3. HRMS (ESI) $\mathrm{m} / \mathrm{z}$ calcd for $\mathrm{C}_{17} \mathrm{H}_{23} \mathrm{~N}_{2} \mathrm{O}[\mathrm{M}+\mathrm{H}]^{+} 271.1805$, found 271.1807.

\subsubsection{4b-Methyl-4b,12-dihydroisoindolo[1,2-b]quinazolin-10(5H)-one (2d)}

Reaction time: $1 \mathrm{~h}$ (room temperature). White solid $(98.1 \mathrm{mg}, 98 \%)$. TLC: $R_{f} 0.26$ (1:1 hexane/EtOAc). mp: 159.2-161.2 ${ }^{\circ} \mathrm{C} .{ }^{1} \mathrm{H}$ NMR $\left(400 \mathrm{MHz}, \mathrm{CDCl}_{3}\right): \delta 8.00$ (dd, $J=7.8$, $1.6 \mathrm{~Hz}, 1 \mathrm{H}), 7.45-7.32(\mathrm{~m}, 5 \mathrm{H}), 7.02-6.93(\mathrm{~m}, 1 \mathrm{H}), 6.87-6.80(\mathrm{~m}, 1 \mathrm{H}), 5.14(\mathrm{~d}, J=15.8 \mathrm{~Hz}$, $1 \mathrm{H}), 4.76(\mathrm{~d}, J=15.8 \mathrm{~Hz}, 1 \mathrm{H}), 4.49$ (brs, $1 \mathrm{H}), 1.66(\mathrm{~s}, 3 \mathrm{H}) .{ }^{13} \mathrm{C} \mathrm{NMR}\left(100 \mathrm{MHz}, \mathrm{CDCl}_{3}\right): \delta$ $161.4,144.7,143.3,135.8,133.5,129.2,128.6,128.3,123.7,120.8,120.7,118.8,117.1,78.7,50.1$, 27.5. HRMS (ESI) $\mathrm{m} / \mathrm{z}$ calcd for $\mathrm{C}_{16} \mathrm{H}_{15} \mathrm{~N}_{2} \mathrm{O}[\mathrm{M}+\mathrm{H}]^{+} 251.1179$, found 251.1182.

3.2.5. 3a-Ethyl-2,2-dimethyl-2,3,3a,4-tetrahydropyrrolo[2,1-b]quinazolin-9(1H)-one (2e)

Reaction time: $24 \mathrm{~h},\left(60{ }^{\circ} \mathrm{C}\right)$. White solid $(77.8 \mathrm{mg}, 80 \%)$. TLC: $R_{f} 0.18$ (3:1 hexanes/EtOAc). mp: 44.2-46.2 ${ }^{\circ} \mathrm{C} .{ }^{1} \mathrm{H} \mathrm{NMR}\left(400 \mathrm{MHz}, \mathrm{CDCl}_{3}\right): \delta 7.87(\mathrm{dd}, J=7.8,1.6 \mathrm{~Hz}$, $1 \mathrm{H}), 7.31-7.22(\mathrm{~m}, 1 \mathrm{H}), 6.84(\mathrm{ddd}, J=7.8,7.3,1.1 \mathrm{~Hz}, 1 \mathrm{H}), 6.65-6.58(\mathrm{~m}, 1 \mathrm{H}), 4.37$ (brs, $1 \mathrm{H})$, $3.99(\mathrm{dd}, J=11.6,1.8 \mathrm{~Hz}, 1 \mathrm{H}), 3.10(\mathrm{dd}, J=11.6,0.8 \mathrm{~Hz}, 1 \mathrm{H}), 2.01(\mathrm{~d}, J=13.2 \mathrm{~Hz}, 1 \mathrm{H}), 1.94$ $(\mathrm{dq}, J=13.6,7.4 \mathrm{~Hz}, 1 \mathrm{H}), 1.84(\mathrm{dd}, J=13.2,1.7 \mathrm{~Hz}, 1 \mathrm{H}), 1.66(\mathrm{dq}, J=13.6,7.4 \mathrm{~Hz}, 1 \mathrm{H}), 1.21$ $(\mathrm{s}, 3 \mathrm{H}), 1.18(\mathrm{~s}, 3 \mathrm{H}), 0.86(\mathrm{t}, J=7.4 \mathrm{~Hz}, 3 \mathrm{H}) .{ }^{13} \mathrm{C} \mathrm{NMR}\left(100 \mathrm{MHz}, \mathrm{CDCl}_{3}\right): \delta 161.4,145.4$, 133.2, 128.6, 119.2, 117.1, 114.9, 78.8, 58.4, 52.2, 37.7, 31.9, 28.1, 26.9, 8.5. HRMS (ESI) m/z calcd for $\mathrm{C}_{15} \mathrm{H}_{21} \mathrm{~N}_{2} \mathrm{O}[\mathrm{M}+\mathrm{H}]^{+} 245.1648$, found 245.1649 .

\subsubsection{5a-Phenyl-5,5a,6,7,8,9-hexahydro-11H-pyrido[2,1-b]quinazolin-11-one (2f)}

Reaction time: $4 \mathrm{~h}\left(120^{\circ} \mathrm{C}\right)$. White solid $(11.5 \mathrm{mg}, 10 \%)$. The reaction mixture was irradiated with microwave in a $5 \mathrm{~mL}$ microwave process vial. TLC: $R_{f} 0.15$ (2:1 hexanes/EtOAc). mp: 151.1-153.1 ${ }^{\circ} \mathrm{C} .{ }^{1} \mathrm{H}$ NMR $\left(400 \mathrm{MHz} \mathrm{CDCl}_{3}\right): \delta 7.94(\mathrm{dd}, J=7.7,1.6 \mathrm{~Hz}, 1 \mathrm{H}), 7.40-7.27$ $(\mathrm{m}, 4 \mathrm{H}), 7.12-7.04(\mathrm{~m}, 2 \mathrm{H}), 6.90(\mathrm{td}, J=7.5,1.0 \mathrm{~Hz}, 1 \mathrm{H}), 6.66(\mathrm{ddd}, J=8.0,1.1,0.5 \mathrm{~Hz}$, $1 \mathrm{H}), 4.52$ (brs, 1H), 3.95-3.83 (m, 1H), 3.72-3.61 (m, 1H), $3.18(\mathrm{dd}, J=13.3,1.3 \mathrm{~Hz}, 1 \mathrm{H})$, $2.79(\mathrm{~d}, \mathrm{~J}=13.3 \mathrm{~Hz}, 1 \mathrm{H}), 2.38-2.29(\mathrm{~m}, 1 \mathrm{H}), 2.14-2.01(\mathrm{~m}, 1 \mathrm{H}), 2.01-1.82(\mathrm{~m}, 2 \mathrm{H}) .{ }^{13} \mathrm{C}$ NMR $\left(100 \mathrm{MHz}, \mathrm{CDCl}_{3}\right): \delta 161.6,145.3,136.2,133.6,130.4,128.8,128.6,127.3,119.6,117.6,115.1$, $77.8,45.1,41.9,36.8,21.1$. HRMS (ESI) $\mathrm{m} / \mathrm{z}$ calcd for $\mathrm{C}_{18} \mathrm{H}_{19} \mathrm{~N}_{2} \mathrm{O}[\mathrm{M}+\mathrm{H}]^{+} 279.1492$, found 279.1494 .

Ketone 3f: Ivory solid (6.8 mg, 6\%). TLC: Rf 0.50 (1:1 hexane/EtOAc). ${ }^{1} \mathrm{H}$ NMR $\left(400 \mathrm{MHz}, \mathrm{CDCl}_{3}\right): \delta 8.01-7.93(\mathrm{~m}, 2 \mathrm{H}), 7.61-7.52(\mathrm{~m}, 1 \mathrm{H}), 7.51-7.42(\mathrm{~m}, 2 \mathrm{H}), 7.36(\mathrm{dd}$, $J=7.8,1.5 \mathrm{~Hz}, 1 \mathrm{H}), 7.20(\mathrm{ddd}, J=8.5,7.2,1.5 \mathrm{~Hz}, 1 \mathrm{H}), 6.71-6.62(\mathrm{~m}, 2 \mathrm{H}), 6.30(\mathrm{~s}, 1 \mathrm{H}), 5.50$ $(\mathrm{s}, 2 \mathrm{H}), 3.46(\mathrm{td}, J=6.8,5.7 \mathrm{~Hz}, 2 \mathrm{H}), 3.06(\mathrm{t}, J=6.8 \mathrm{~Hz}, 2 \mathrm{H}), 1.92-1.80(\mathrm{~m}, 2 \mathrm{H}), 1.76-1.65$ (m, 2H). ${ }^{13} \mathrm{C}$ NMR $\left(100 \mathrm{MHz}, \mathrm{CDCl}_{3}\right): \delta 200.3,169.5,148.8,137.0,133.3,132.3,128.8,128.2$, $127.3,117.4,116.8,116.4,39.4,38.1,29.3,21.3$. HRMS (ESI) m/z calcd for $\mathrm{C}_{18} \mathrm{H}_{21} \mathrm{~N}_{2} \mathrm{O}_{2}$ $[\mathrm{M}+\mathrm{H}]^{+}$297.1598, found 297.1601.

\subsubsection{5a-Methyl-5,5a,6,7,8,9-hexahydro-11H-pyrido[2,1-b]quinazolin-11-one (2g)}

Reaction time: $1 \mathrm{~h}\left(120^{\circ} \mathrm{C}\right)$. White solid $(25.7 \mathrm{mg}, 30 \%)$. The reaction mixture was irradiated with microwave in a $5 \mathrm{~mL}$ microwave process vial. TLC: $R_{f} 0.20$ (2:1 hexanes/EtOAc). mp: $139.8-141.8{ }^{\circ} \mathrm{C} .{ }^{1} \mathrm{H}$ NMR $\left(400 \mathrm{MHz}, \mathrm{CDCl}_{3}\right): \delta 7.90(\mathrm{dd}, J=7.9,1.6 \mathrm{~Hz}, 1 \mathrm{H}), 7.31-7.22$ $(\mathrm{m}, 1 \mathrm{H}), 6.80(\mathrm{ddd}, J=7.9,7.3,1.0 \mathrm{~Hz}, 1 \mathrm{H}), 6.56(\mathrm{~d}, J=7.9,1.0 \mathrm{~Hz}, 1 \mathrm{H}), 4.54(\mathrm{~m}, 1 \mathrm{H}), 4.03$ (brs, 1H), $2.74(\mathrm{~m}, 1 \mathrm{H}), 1.98-1.89(\mathrm{~m}, 1 \mathrm{H}), 1.87-1.81(\mathrm{~m}, 3 \mathrm{H}), 1.65-1.50(\mathrm{~m}, 2 \mathrm{H}), 1.47(\mathrm{~s}, 3 \mathrm{H})$. ${ }^{13} \mathrm{C} \mathrm{NMR}\left(100 \mathrm{MHz} \mathrm{CDCl}_{3}\right): \delta 163.9,144.7,133.6,128.9,118.9,115.8,114.3,70.7,39.2,38.6$, 24.4, 22.3, 20.6. HRMS (ESI) $\mathrm{m} / \mathrm{z}$ calcd for $\mathrm{C}_{13} \mathrm{H}_{17} \mathrm{~N}_{2} \mathrm{O}[\mathrm{M}+\mathrm{H}]^{+}$217.1335, found 217.1335.

Ketone 3g: Colorless liquid (14.0 mg, 15\%). TLC: $R_{f} 0.15$ (1.5:1 hexanes/EtOAc). ${ }^{1} \mathrm{H}$ $\operatorname{NMR}\left(400 \mathrm{MHz}, \mathrm{CDCl}_{3}\right): \delta 7.34(\mathrm{dd}, J=7.9,1.5 \mathrm{~Hz}, 1 \mathrm{H}), 7.20(\mathrm{ddd}, J=8.5,7.2,1.5 \mathrm{~Hz}, 1 \mathrm{H})$, 6.71-6.61 (m, 2H), $6.24(\mathrm{~s}, 1 \mathrm{H}), 5.50(\mathrm{~s}, 2 \mathrm{H}), 3.40(\mathrm{td}, J=6.6,5.6 \mathrm{~Hz}, 2 \mathrm{H}), 2.51(\mathrm{t}, J=6.8 \mathrm{~Hz}$, $2 \mathrm{H}), 2.15(\mathrm{~s}, 3 \mathrm{H}), 1.73-1.59(\mathrm{~m}, 4 \mathrm{H}) .{ }^{13} \mathrm{C} \mathrm{NMR}\left(100 \mathrm{MHz}, \mathrm{CDCl}_{3}\right): \delta 163.9,144.7,133.6,128.9$, 
$118.9,115.8,114.3,70.7,39.7,38.6,24.4,22.3,20.6$. HRMS (ESI) $\mathrm{m} / \mathrm{z}$ calcd for $\mathrm{C}_{13} \mathrm{H}_{19} \mathrm{~N}_{2} \mathrm{O}_{2}$ $[\mathrm{M}+\mathrm{H}]^{+}$235.1441, found 235.1442.

3.2.8. 2-methoxy-5a-methyl-5,5a,6,7,8,9-hexahydro-11H-pyrido[2,1-b]quinazolin-11-one (2h)

Reaction time: $2.5 \mathrm{~h}\left(120^{\circ} \mathrm{C}\right)$. A total of $754 \mu \mathrm{mol}$ of $\mathbf{1 h}$ was used. White solid (175 mg, $94 \%$ ). The reaction mixture was irradiated with microwave in a $5 \mathrm{~mL}$ microwave process vial. TLC: $R_{f} 0.30$ (2:1 hexanes/EtOAc). mp: $146.7-147.8^{\circ} \mathrm{C} .{ }^{1} \mathrm{H}$ NMR $\left(400 \mathrm{MHz}, \mathrm{DMSO}-d_{6}\right)$ : $\delta 7.14(\mathrm{~d}, J=3.0 \mathrm{~Hz}, 1 \mathrm{H}), 6.91(\mathrm{dd}, J=8.7,3.0 \mathrm{~Hz}, 1 \mathrm{H}), 6.61(\mathrm{~d}, J=8.7 \mathrm{~Hz}, 1 \mathrm{H}), 6.34(\mathrm{~s}, 1 \mathrm{H})$, $4.35-4.13(\mathrm{~m}, 1 \mathrm{H}), 3.67(\mathrm{~s}, 3 \mathrm{H}), 2.65(\mathrm{td}, J=13.4,3.5 \mathrm{~Hz}, 1 \mathrm{H}), 1.90-1.65(\mathrm{~m}, 4 \mathrm{H}), 1.62-1.34$ $(\mathrm{m}, 2 \mathrm{H}), 1.32(\mathrm{~s}, 3 \mathrm{H}) .{ }^{13} \mathrm{C}$ NMR (100 MHz, DMSO- $\left.d_{6}\right): \delta 162.7,151.1,140.4,121.3,115.7$, $114.5,110.3,70.4,55.3,38.0,37.8,24.0,20.9,19.8$. HRMS (EI) $\mathrm{m} / \mathrm{z}$ calcd for $\mathrm{C}_{14} \mathrm{H}_{18} \mathrm{~N}_{2} \mathrm{O}_{2}$ $[\mathrm{M}]^{+} 246.1368$, found 246.1364 .

\subsubsection{2,3-dimethoxy-5a-methyl-5,5a,6,7,8,9-hexahydro-11H-pyrido[2,1-b]quinazolin-11-one (2i)}

Reaction time: $1.25 \mathrm{~h}\left(120^{\circ} \mathrm{C}\right)$. A total of $719 \mu \mathrm{mol}$ of $1 \mathrm{i}$ was used. White solid $(167 \mathrm{mg}$, $84 \%)$. The reaction mixture was irradiated with microwave in a $5 \mathrm{~mL}$ microwave process vial. TLC: $R_{f} 0.30$ (1:1 hexanes/EtOAc). mp: $195.5-196.7^{\circ} \mathrm{C} .{ }^{1} \mathrm{H}$ NMR $\left(400 \mathrm{MHz}, \mathrm{DMSO}-d_{6}\right)$ : $\delta 7.09(\mathrm{~s}, 1 \mathrm{H}), 6.40(\mathrm{~s}, 1 \mathrm{H}), 6.22(\mathrm{~s}, 1 \mathrm{H}), 4.22(\mathrm{dd}, J=12.4,4.3 \mathrm{~Hz}, 1 \mathrm{H}), 3.74(\mathrm{~s}, 3 \mathrm{H}), 3.66(\mathrm{~s}, 3 \mathrm{H})$, $2.61(\mathrm{td}, J=13.5,3.3 \mathrm{~Hz}, 1 \mathrm{H}), 1.87-1.65(\mathrm{~m}, 4 \mathrm{H}), 1.62-1.44(\mathrm{~m}, 1 \mathrm{H}), 1.37(\mathrm{td}, J=8.8,4.3 \mathrm{~Hz}$, 1H), 1.32 (s, 3H). ${ }^{13} \mathrm{C}$ NMR (100 MHz, DMSO- $\left.d_{6}\right): \delta 162.8,153.9,141.8,141.1,110.4,105.5$, 97.5, 70.4, 55.9, 55.3, 38.1, 37.7, 24.1, 20.6, 19.9. HRMS (ESI) $\mathrm{m} / \mathrm{z}$ calcd for $\mathrm{C}_{15} \mathrm{H}_{21} \mathrm{~N}_{2} \mathrm{O}_{3}$ $[\mathrm{M}+\mathrm{H}]^{+}$277.1547, found 277.1546.

3.2.10. 5a,8,8-Trimethyl-5,5a,6,7,8,9-hexahydro-11H-pyrido[2,1-b]quinazolin-11-one (2j)

Reaction time: $20 \mathrm{~min}\left(120^{\circ} \mathrm{C}\right)$. White solid $(55.2 \mathrm{mg}, 57 \%)$. The reaction mixture was irradiated with microwave in a $5 \mathrm{~mL}$ microwave process vial. TLC: $R_{f} 0.20$ (2:1 hexanes/EtOAc). mp: $121.6-123.6{ }^{\circ} \mathrm{C} .{ }^{1} \mathrm{H} \mathrm{NMR}\left(400 \mathrm{MHz}, \mathrm{CDCl}_{3}\right): \delta 7.91$ (dd, $J=7.8,1.6$ $\mathrm{Hz}, 1 \mathrm{H}), 7.27(\mathrm{~m}, 1 \mathrm{H}), 6.81(\mathrm{td}, J=7.5,1.1 \mathrm{~Hz}, 1 \mathrm{H}), 6.57(\mathrm{dd}, J=8.1,1.1 \mathrm{~Hz}, 1 \mathrm{H}), 4.22(\mathrm{dd}$, $J=13.7,1.9 \mathrm{~Hz}, 1 \mathrm{H}), 4.03(\mathrm{brs}, 1 \mathrm{H}), 2.49(\mathrm{dd}, J=13.7,0.8 \mathrm{~Hz}, 1 \mathrm{H}), 2.13(\mathrm{td}, J=12.7,5.5 \mathrm{~Hz}$, $1 \mathrm{H}), 1.72(\mathrm{dt}, J=12.7,4.1 \mathrm{~Hz}, 1 \mathrm{H}), 1.54-1.45(\mathrm{~m}, 2 \mathrm{H}), 1.44(\mathrm{~d}, J=0.8 \mathrm{~Hz}, 3 \mathrm{H}), 1.03(\mathrm{~s}, 3 \mathrm{H})$, $1.02(\mathrm{~s}, 3 \mathrm{H}) .{ }^{13} \mathrm{C}$ NMR $\left(100 \mathrm{MHz}, \mathrm{CDCl}_{3}\right): \delta 164.0,144.7,133.6,128.9,118.9,114.3,77.4$, $70.3,49.1,35.7,33.8,30.1,29.0,23.9,22.4$. HRMS (ESI) $\mathrm{m} / \mathrm{z}$ calcd for $\mathrm{C}_{15} \mathrm{H}_{21} \mathrm{~N}_{2} \mathrm{O}[\mathrm{M}+\mathrm{H}]^{+}$ 245.1648 , found 245.1651 .

\subsubsection{3a-Methyl-5,6,13,13a-tetrahydro-8H-isoquinolino[1,2-b]quinazolin-8-one (2k)}

Reaction time: $1 \mathrm{~h}\left(120^{\circ} \mathrm{C}\right)$. White solid $(64.6 \mathrm{mg}, 61 \%)$. The reaction mixture was irradiated with microwave in a $5 \mathrm{~mL}$ microwave process vial. TLC: $R_{f} 0.20$ (2:1 hexanes/EtOAc). mp: 187.3-189.3 ${ }^{\circ} \mathrm{C} .{ }^{1} \mathrm{H}$ NMR $\left(400 \mathrm{MHz}, \mathrm{CDCl}_{3}\right): \delta 8.01(\mathrm{dd}, J=7.9,1.5 \mathrm{~Hz}, 1 \mathrm{H}), 7.42(\mathrm{dd}$, $J=7.9,1.5 \mathrm{~Hz}, 1 \mathrm{H}), 7.39-7.28(\mathrm{~m}, 3 \mathrm{H}), 7.21(\mathrm{dd}, J=7.5,1.6 \mathrm{~Hz}, 1 \mathrm{H}), 6.92(\mathrm{ddd}, J=8.2,7.3$, $1.1 \mathrm{~Hz}, 1 \mathrm{H}), 6.76-6.70(\mathrm{~m}, 1 \mathrm{H}), 5.07-4.94(\mathrm{~m}, 1 \mathrm{H}), 4.36$ (brs, $1 \mathrm{H}), 3.12-2.95(\mathrm{~m}, 2 \mathrm{H}), 2.89-2.76$ $(\mathrm{m}, 1 \mathrm{H}), 1.78(\mathrm{~s}, 3 \mathrm{H}) .{ }^{13} \mathrm{C}$ NMR $\left(100 \mathrm{MHz}, \mathrm{CDCl}_{3}\right): \delta 162.7,144.4,138.9,134.6,133.6,129.6$, 129.0, 128.2, 127.5, 124.6, 120.0, 117.3, 116.2, 71.5, 36.0, 29.2, 26.3. HRMS (ESI) $\mathrm{m} / \mathrm{z}$ calcd for $\mathrm{C}_{17} \mathrm{H}_{17} \mathrm{~N}_{2} \mathrm{O}[\mathrm{M}+\mathrm{H}]^{+}$265.1335, found 265.1336.

3.2.12. 5a,9,9-Trimethyl-5a,6,7,8,9,10-hexahydroazepino[2,1-b]quinazolin-12(5H)-one (21)

Reaction time: $1 \mathrm{~h}\left(120^{\circ} \mathrm{C}\right)$. White solid $(31.5 \mathrm{mg}, 31 \%)$. The reaction mixture was irradiated with microwave in a $5 \mathrm{~mL}$ microwave process vial. TLC: $R_{f} 0.25$ (4:1 hexanes/EtOAc). mp: 177.9-179.9 ${ }^{\circ} \mathrm{C} .{ }^{1} \mathrm{H}$ NMR $\left(400 \mathrm{MHz}, \mathrm{CDCl}_{3}\right): \delta 7.87(\mathrm{dd}, J=7.5,1.6 \mathrm{~Hz}, 1 \mathrm{H}), 7.24(\mathrm{ddd}$, $J=8.0,7.5,1.6 \mathrm{~Hz}, 1 \mathrm{H}), 6.78(\mathrm{td}, J=7.5,1.0 . \mathrm{Hz}, 1 \mathrm{H}), 6.59(\mathrm{dd}, J=8.0,1.0 \mathrm{~Hz}, 1 \mathrm{H}), 4.29(\mathrm{dd}$, $J=14.1,2.1 \mathrm{~Hz}, 1 \mathrm{H}), 4.10(\mathrm{brs}, 1 \mathrm{H}), 2.62(\mathrm{~d}, J=14.1 \mathrm{~Hz}, 1 \mathrm{H}), 2.21-2.04(\mathrm{~m}, 1 \mathrm{H}), 1.98-1.75$ $(\mathrm{m}, 2 \mathrm{H}), 1.59-1.43(\mathrm{~m}, 2 \mathrm{H}), 1.40(\mathrm{~s}, 3 \mathrm{H}), 1.29-1.14(\mathrm{~m}, 1 \mathrm{H}), 0.96(\mathrm{~s}, 3 \mathrm{H}), 0.91(\mathrm{~s}, 3 \mathrm{H}) .{ }^{13} \mathrm{C}$ NMR (100 MHz, CDCl $)$ : $\delta$ 164.0, 145.6, 133.3, 129.1, 118.9, 115.8, 114.5, 74.3, 49.3, 44.8, 43.8, 35.5, 29.7, 25.6, 23.4, 19.2. HRMS (ESI) $\mathrm{m} / \mathrm{z}$ calcd for $\mathrm{C}_{16} \mathrm{H}_{23} \mathrm{~N}_{2} \mathrm{O}[\mathrm{M}+\mathrm{H}]^{+} 259.1805$, found 259.1806. 
Ketone 31: Colorless liquid (18.8 mg, 17\%). TLC: $R_{f} 0.08$ (4:1 hexanes/EtOAc). ${ }^{1} \mathrm{H}$ NMR $\left(400 \mathrm{MHz}, \mathrm{CDCl}_{3}\right): \delta 7.43(\mathrm{dd}, J=8.1,1.5 \mathrm{~Hz}, 1 \mathrm{H}), 7.23-7.15(\mathrm{~m}, 1 \mathrm{H}), 6.71-6.62(\mathrm{~m}$, $2 \mathrm{H}), 6.49(\mathrm{~s}, 1 \mathrm{H}), 5.48(\mathrm{~s}, 2 \mathrm{H}), 3.28(\mathrm{~d}, J=6.4 \mathrm{~Hz}, 2 \mathrm{H}), 2.44(\mathrm{t}, J=6.7 \mathrm{~Hz}, 2 \mathrm{H}), 2.13(\mathrm{~s}, 3 \mathrm{H})$, 1.62-1.50 (m, 2H), 1.23-1.16 (m, 2H), $0.92(\mathrm{~s}, 6 \mathrm{H}) .{ }^{13} \mathrm{C} \mathrm{NMR}\left(100 \mathrm{MHz}, \mathrm{CDCl}_{3}\right)$ : $\delta 209.4$, 169.6, 148.8, 132.2, 127.3, 117.3, 116.8, 116.7, 48.1, 43.8, 39.0, 34.8, 30.2, 25.4, 17.9. HRMS (ESI) $\mathrm{m} / \mathrm{z}$ calcd for $\mathrm{C}_{16} \mathrm{H}_{25} \mathrm{~N}_{2} \mathrm{O}_{2}[\mathrm{M}+\mathrm{H}]+277.1911$, found 277.1913 .

\subsubsection{8-Chloro-4b-methyl-4b,12-dihydroisoindolo[1,2-b]quinazolin-10(5H)-one (2m)}

Reaction time: $30 \mathrm{~min}$ (room temperature). White solid (109.8 mg, 96\%). TLC: $R_{f}$ 0.34 (2:1 hexane/EtOAc). mp: $207.3-209.2{ }^{\circ} \mathrm{C} .{ }^{1} \mathrm{H}$ NMR $\left(400 \mathrm{MHz}, \mathrm{CDCl}_{3}\right): \delta 7.95(\mathrm{~d}$, $J=2.5 \mathrm{~Hz}, 1 \mathrm{H}), 7.45-7.35(\mathrm{~m}, 4 \mathrm{H}), 7.30(\mathrm{dd}, J=8.5,2.5 \mathrm{~Hz}, 1 \mathrm{H}), 6.80(\mathrm{~d}, J=8.5 \mathrm{~Hz}, 1 \mathrm{H})$, $5.10(\mathrm{~d}, J=15.8 \mathrm{~Hz}, 1 \mathrm{H}), 4.74(\mathrm{~d}, J=15.8 \mathrm{~Hz}, 1 \mathrm{H}), 4.67(\mathrm{brs}, 1 \mathrm{H}), 1.65(\mathrm{~s}, 3 \mathrm{H}) .{ }^{13} \mathrm{C}$ NMR $(100$ $\left.\mathrm{MHz}, \mathrm{CDCl}_{3}\right): \delta 160.3,143.2,142.8,135.5,133.5,129.3,128.4,128.2,125.8,123.7,120.8,119.8$, 118.6, 78.9, 50.1, 27.5. HRMS (ESI) $\mathrm{m} / \mathrm{z}$ calcd for $\mathrm{C}_{16} \mathrm{H}_{14} \mathrm{ClN}_{2} \mathrm{O}[\mathrm{M}+\mathrm{H}]^{+} 285.0789$, found 285.0789 .

\subsubsection{7-Chloro-4b-methyl-4b,12-dihydroisoindolo[1,2-b]quinazolin-10(5H)-one (2n)}

Reaction time: $30 \mathrm{~min}$ (room temperature). White solid (110.7 mg, 97\%). TLC: $R_{f} 0.28$ (2:1 hexane/EtOAc). mp: $232.3-233.7^{\circ} \mathrm{C} .{ }^{1} \mathrm{H}$ NMR $\left(400 \mathrm{MHz}, \mathrm{CDCl}_{3}\right): \delta 7.92(\mathrm{~d}, J=8.3 \mathrm{~Hz}$, $1 \mathrm{H}), 7.44-7.39(\mathrm{~m}, 3 \mathrm{H}), 7.39-7.33(\mathrm{~m}, 1 \mathrm{H}), 6.92(\mathrm{dd}, J=8.3,1.9 \mathrm{~Hz}, 1 \mathrm{H}), 6.83(\mathrm{~d}, J=1.9 \mathrm{~Hz}$, $1 \mathrm{H}), 5.12(\mathrm{~d}, J=16.0 \mathrm{~Hz}, 1 \mathrm{H}), 4.74(\mathrm{~d}, J=16.0 \mathrm{~Hz}, 1 \mathrm{H}), 4.57(\mathrm{brs}, 1 \mathrm{H}), 1.67(\mathrm{~s}, 3 \mathrm{H}) .{ }^{13} \mathrm{C}$ NMR $\left(100 \mathrm{MHz}, \mathrm{CDCl}_{3}\right): \delta 160.2,143.6,142.8,136.3,135.5,131.2,129.3,128.4,123.7,120.8,120.0$, $118.8,112.7,78.9,50.1,27.5$. HRMS (ESI) $\mathrm{m} / \mathrm{z}$ calcd for $\mathrm{C}_{16} \mathrm{H}_{14} \mathrm{ClN}_{2} \mathrm{O}[\mathrm{M}+\mathrm{H}]^{+} 285.0789$, found 285.0790 .

\subsubsection{8-Bromo-4b-methyl-4b,12-dihydroisoindolo[1,2-b]quinazolin-10(5H)-one (2o)}

Reaction time: $2 \mathrm{~h}$ (room temperature). White solid (121.6 mg, 92\%). TLC: $R_{f} 0.28$ (2:1 hexane/EtOAc). mp: $213.1-215.1^{\circ} \mathrm{C} .{ }^{1} \mathrm{H}$ NMR $\left(400 \mathrm{MHz}, \mathrm{CDCl}_{3}\right): \delta 8.11(\mathrm{~d}, J=2.3 \mathrm{~Hz}$, $1 \mathrm{H}), 7.45(\mathrm{dd}, J=8.5,2.4 \mathrm{~Hz}, 1 \mathrm{H}), 7.42-7.39(\mathrm{~m}, 3 \mathrm{H}), 7.39-7.35(\mathrm{~m}, 1 \mathrm{H}), 6.73(\mathrm{~d}, J=8.5 \mathrm{~Hz}$, $1 \mathrm{H}), 5.12(\mathrm{~d}, J=16.0 \mathrm{~Hz}, 1 \mathrm{H}), 4.75(\mathrm{~d}, J=16.0 \mathrm{~Hz}, 1 \mathrm{H}), 4.48(\mathrm{brs}, 1 \mathrm{H}), 1.66(\mathrm{~s}, 3 \mathrm{H}) .{ }^{13} \mathrm{C}$ NMR $\left(100 \mathrm{MHz}, \mathrm{CDCl}_{3}\right): \delta 160.2,143.6,142.8,136.3,135.5,131.2,129.3,128.4,123.7,120.8,120.0$, $118.8,112.7,78.9,50.1,27.5$. HRMS (ESI) $\mathrm{m} / \mathrm{z}$ calcd for $\mathrm{C}_{16} \mathrm{H}_{14} \mathrm{BrN}_{2} \mathrm{O}[\mathrm{M}+\mathrm{H}]^{+} 329.0284$, found 329.0285 .

\subsubsection{4b-methyl-7-nitro-4b,12-dihydroisoindolo[1,2-b]quinazolin-10(5H)-one (2p)}

Reaction time: $30 \mathrm{~min}$ (room temperature). Yellow solid (114.8 mg, 97\%). TLC: $R_{f} 0.25$ (1:1 hexane/EtOAc). mp: $279.1-281.4{ }^{\circ} \mathrm{C} .{ }^{1} \mathrm{H}$ NMR $\left(400 \mathrm{MHz}\right.$, DMSO- $\left.d_{6}\right): \delta 8.15$ (brs, $\left.1 \mathrm{H}\right)$, $7.93(\mathrm{~d}, J=8.5 \mathrm{~Hz}, 1 \mathrm{H}), 7.68(\mathrm{~d}, J=2.3 \mathrm{~Hz}, 1 \mathrm{H}), 7.62-7.53(\mathrm{~m}, 2 \mathrm{H}), 7.51-7.36(\mathrm{~m}, 3 \mathrm{H}), 4.99(\mathrm{~d}$, $J=16.0 \mathrm{~Hz}, 1 \mathrm{H}), 4.70(\mathrm{~d}, J=16.0 \mathrm{~Hz}, 1 \mathrm{H}), 1.58(\mathrm{~s}, 3 \mathrm{H}) .{ }^{13} \mathrm{C}$ NMR $\left(100 \mathrm{MHz}, \mathrm{DMSO}-d_{6}\right): \delta$ 158.5, 150.7, 146.9, 142.8, 134.7, 129.2, 128.7, 128.0, 123.5, 121.3, 120.0, 111.9, 109.8, 78.5, 49.5, 27.1. HRMS (ESI) $\mathrm{m} / \mathrm{z}$ calcd for $\mathrm{C}_{16} \mathrm{H}_{14} \mathrm{~N}_{3} \mathrm{O}_{3}[\mathrm{M}+\mathrm{H}]^{+} 296.1030$, found 296.1031 .

\subsubsection{4b,8-Dimethyl-4b,12-dihydroisoindolo[1,2-b]quinazolin-10(5H)-one (2q)}

Reaction time: $8 \mathrm{~h}$ (room temperature). White solid (96.2 mg, 91\%). TLC: $R_{f} 0.14$ (2:1 hexane/EtOAc). mp: $191.2-192.7^{\circ} \mathrm{C} .{ }^{1} \mathrm{H}$ NMR $\left(400 \mathrm{MHz}, \mathrm{CDCl}_{3}\right): \delta 7.76(\mathrm{~d}, J=1.6 \mathrm{~Hz}$, $1 \mathrm{H}), 7.49-7.42(\mathrm{~m}, 1 \mathrm{H}), 7.39-7.28(\mathrm{~m}, 3 \mathrm{H}), 7.16(\mathrm{dd}, J=8.2,2.0 \mathrm{~Hz}, 1 \mathrm{H}), 6.84(\mathrm{~d}, J=8.2 \mathrm{~Hz}$, 1H), 5.11 (brs, 1H), $5.06(\mathrm{~d}, J=15.8 \mathrm{~Hz}, 1 \mathrm{H}), 4.69(\mathrm{~d}, J=15.8 \mathrm{~Hz}, 1 \mathrm{H}), 2.29(\mathrm{~s}, 3 \mathrm{H}), 1.63$ (s, 3H). ${ }^{13} \mathrm{C} \mathrm{NMR}\left(100 \mathrm{MHz}, \mathrm{CDCl}_{3}\right): \delta 161.5,143.2,142.1,135.6,134.4,130.4,129.0,128.4$, $128.2,123.5,121.2,118.9,117.8,78.9,49.9,27.0,20.7$. HRMS (ESI) $\mathrm{m} / \mathrm{z}$ calcd for $\mathrm{C}_{17} \mathrm{H}_{17} \mathrm{~N}_{2} \mathrm{O}$ $[\mathrm{M}+\mathrm{H}]^{+}$265.1335, found 265.1335.

\subsubsection{8-Methoxy-4b-methyl-4b,12-dihydroisoindolo[1,2-b]quinazolin-10(5H)-one (2r)}

Reaction time: $24 \mathrm{~h}$ (room temperature). White solid ( $84.7 \mathrm{mg}, 76 \%)$. TLC: $R_{f} 0.26$ (1:2 hexane/EtOAc). mp: $192.5-193.8^{\circ} \mathrm{C} .{ }^{1} \mathrm{H} \mathrm{NMR}\left(400 \mathrm{MHz}, \mathrm{CDCl}_{3}\right): \delta 7.54(\mathrm{~d}, J=2.9 \mathrm{~Hz}, 1 \mathrm{H})$, 
7.44-7.37 (m, 4H), $7.01(\mathrm{dd}, J=8.7,3.0 \mathrm{~Hz}, 1 \mathrm{H}), 6.90(\mathrm{~d}, J=8.7 \mathrm{~Hz}, 1 \mathrm{H}), 5.12(\mathrm{~d}, J=15.9 \mathrm{~Hz}$, $1 \mathrm{H}), 4.75(\mathrm{~d}, J=15.9 \mathrm{~Hz}, 1 \mathrm{H}), 3.85(\mathrm{~s}, 3 \mathrm{H}), 1.61(\mathrm{~s}, 3 \mathrm{H}) .{ }^{13} \mathrm{C} \mathrm{NMR}\left(100 \mathrm{MHz}, \mathrm{CDCl}_{3}\right): \delta 161.2$, 155.0, 143.4, 137.9, 135.9, 129.2, 128.3, 123.6, 121.5, 121.4, 121.3, 120.9, 110.7, 79.1, 55.9, 49.8, 27.1. HRMS (ESI) $\mathrm{m} / \mathrm{z}$ calcd for $\mathrm{C}_{17} \mathrm{H}_{16} \mathrm{~N}_{2} \mathrm{NaO}_{2}[\mathrm{M}+\mathrm{Na}]^{+}$303.1104, found 303.1107.

3.2.19. 7,8-dimethoxy-4b-methyl-4b,12-dihydroisoindolo[1,2-b]quinazolin-10(5H)-one (2s)

Reaction time: $3 \mathrm{~h}$ (room temperature). White solid $(88.9 \mathrm{mg}, 72 \%)$. TLC: $R_{f} 0.22(1: 1$ hexane/EtOAc). mp: 154.6-156.1 ${ }^{\circ} \mathrm{C} .{ }^{1} \mathrm{H}$ NMR (400 MHz, CD 3 OD): $\delta 7.55-7.49$ (m, 1H), $7.46-7.38(\mathrm{~m}, 3 \mathrm{H}), 7.33(\mathrm{~s}, 1 \mathrm{H}), 6.50(\mathrm{~s}, 1 \mathrm{H}), 5.01(\mathrm{~d}, J=15.9 \mathrm{~Hz}, 1 \mathrm{H}), 4.70(\mathrm{~d}, J=15.9 \mathrm{~Hz}, 1 \mathrm{H})$, 3.87 (s, 3H), 3.82 (s, 3H), 1.62 (s, 3H). $\left.{ }^{13} \mathrm{C} \mathrm{NMR} \mathrm{(100} \mathrm{MHz,} \mathrm{CD} 3 \mathrm{OD}\right): \delta$ 163.6, 156.3, 144.8, 144.3, 143.8, 136.4, 129.9, 129.2, 124.3, 122.3, 111.4, 109.0, 100.5, 80.4, 57.0, 56.3, 50.7, 26.2. HRMS (ESI) $\mathrm{m} / \mathrm{z}$ calcd for $\mathrm{C}_{18} \mathrm{H}_{19} \mathrm{~N}_{2} \mathrm{O}_{3}[\mathrm{M}+\mathrm{H}]^{+} 311.1390$, found 311.1393 .

\subsubsection{6,7,8-trimethoxy-4b-methyl-4b,12-dihydroisoindolo[1,2-b]quinazolin-10(5H)-one (2t)}

Reaction time: $1 \mathrm{~h}$ (room temperature). White solid $(116.5 \mathrm{mg}, 86 \%)$ TLC: $R_{f} 0.16(1: 1$ hexane/EtOAc). mp: $190.4-192.4{ }^{\circ} \mathrm{C} .{ }^{1} \mathrm{H} \mathrm{NMR}\left(400 \mathrm{MHz}, \mathrm{CDCl}_{3}\right): \delta 7.43$ (dtd, $J=5.7,3.1$, $1.1 \mathrm{~Hz}, 1 \mathrm{H}), 7.41-7.33(\mathrm{~m}, 3 \mathrm{H}), 7.28(\mathrm{~s}, 1 \mathrm{H}), 5.10(\mathrm{~d}, J=15.8 \mathrm{~Hz}, 1 \mathrm{H}), 4.81$ (brs, 1H), 4.74 (d, $J=15.8 \mathrm{~Hz}, 1 \mathrm{H}), 3.94(\mathrm{~s}, 3 \mathrm{H}), 3.88(\mathrm{~s}, 3 \mathrm{H}), 3.86(\mathrm{~s}, 3 \mathrm{H}), 1.64(\mathrm{~s}, 3 \mathrm{H}) .{ }^{13} \mathrm{C} \mathrm{NMR}(100 \mathrm{MHz}$, $\left.\mathrm{CDCl}_{3}\right): \delta 160.9,146.9,146.4,143.1,141.0,135.4,133.7,128.9,128.1,123.4,120.8,112.1$, $105.7,79.0,60.9,60.9,56.3,50.0,26.8$. HRMS (ESI) $\mathrm{m} / \mathrm{z}$ calcd for $\mathrm{C}_{19} \mathrm{H}_{20} \mathrm{~N}_{2} \mathrm{NaO}_{4}[\mathrm{M}+\mathrm{Na}]^{+}$ 363.1315 , found 363.1318 .

\subsubsection{4b,5-dimethyl-4b,12-dihydroisoindolo[1,2-b]quinazolin-10(5H)-one (2u)}

Reaction time: $1.5 \mathrm{~h}\left(60{ }^{\circ} \mathrm{C}\right)$. White solid (102.5 mg, 97\%). TLC: $R_{f} 0.15$ (5:1 hexane/EtOAc). mp: $133.0-134.8^{\circ} \mathrm{C} .{ }^{1} \mathrm{H}$ NMR $\left(400 \mathrm{MHz}, \mathrm{CDCl}_{3}\right): \delta 8.05(\mathrm{dd}, J=7.7,1.4 \mathrm{~Hz}$, 1H), 7.55-7.44 (m, 2H), 7.44-7.38 (m, 3H), 7.14-7.03 (m, 2H), $5.13(\mathrm{~d}, J=15.9 \mathrm{~Hz}, 1 \mathrm{H}), 4.81$ $(\mathrm{d}, J=15.9 \mathrm{~Hz}, 1 \mathrm{H}), 2.74(\mathrm{~s}, 3 \mathrm{H}), 1.54(\mathrm{~s}, 3 \mathrm{H}) .{ }^{13} \mathrm{C} \mathrm{NMR}\left(100 \mathrm{MHz}, \mathrm{CDCl}_{3}\right): \delta 161.5,149.0$, 140.7, 136.4, 133.4, 129.2, 128.1, 127.7, 124.4, 123.7, 122.4, 121.9, 119.7, 83.4, 50.4, 37.1, 25.5. HRMS (ESI) $\mathrm{m} / \mathrm{z}$ calcd for $\mathrm{C}_{17} \mathrm{H}_{16} \mathrm{~N}_{2} \mathrm{NaO}[\mathrm{M}+\mathrm{Na}]^{+} 287.1155$, found 287.1158.

\section{Conclusions}

In conclusion, we developed a gold(I)-catalyzed one-pot cascade process for alkynetethered anthranilamides, which allows facile access to polycyclic dihydroquinazolinones in good to excellent yields under mild reaction conditions. This one-pot cascade methodology is widely applicable to the synthesis of bioactive privileged natural product scaffolds and offers a straightforward approach to increase molecular complexity and structural diversity in a few synthetic steps. The use of this method to construct polycyclic dihydroquinazolinonelike libraries is currently underway in our laboratory.

Supplementary Materials: The following are available online at https: / www.mdpi.com/article / $10.3390 /$ catal11121436/s1, detailed synthetic procedure of $\mathbf{1 a}-\mathbf{u}$ and copies of ${ }^{1} \mathrm{H}$ and ${ }^{13} \mathrm{C}$ NMR spectra of $\mathbf{1 a - u}$ and $\mathbf{2 a - u}$.

Author Contributions: Conceptualization, J.-S.R.; investigation, experimental, and analysis, J.S., Y.S., and J.-S.R.; resources, J.-S.R.; data curation, J.S. and J.-S.R.; writing-original draft preparation, J.-S.R.; writing-review and editing, J.S. and J.-S.R.; supervision, J.-S.R.; project administration, J.-S.R.; funding acquisition, J.-S.R. All authors have read and agreed to the published version of the manuscript.

Funding: This research was supported by the National Research Foundation of Korea (NRF) grant funded by the Korea government (MSIT) (NRF-2018R1A5A2025286), (NRF-2020R1F1A1052496), and (NRF-2021R1A6C101A442).

Acknowledgments: We gratefully acknowledge Drug Development Research Core Center for $400 \mathrm{MHz}$ NMR Spectrometer.

Conflicts of Interest: The authors declare no conflict of interest. 


\section{References}

1. Michael, J.P. Indolizidine and quinolizidine alkaloid. Nat. Prod. Rep. 2002, 19, 719-741. [CrossRef]

2. Yamamoto, Y. Synthesis of heterocycles via transition-metal-catalyzed hydroarylation of alkynes. Chem. Soc. Rev. 2014, 43, 1575-1600. [CrossRef]

3. Deiters, A.; Martin, S.F. Synthesis of oxygen- and nitrogen-containing heterocycles by ring-closing metathesis. Chem. Rev. 2004, 104, 2199-2238. [CrossRef]

4. Khan, I.; Ibrar, A.; Abbas, N.; Saeed, A. Recent advances in the structural library of functionalized quinazoline and quinazolinone scaffolds: Synthetic approaches and multifarious applications. Eur. J. Med. Chem. 2014, 76, 193-244. [CrossRef] [PubMed]

5. Kshirsagar, U.A. Recent developments in the chemistry of quinazolinone alkaloids. Org. Biomol. Chem. 2015, 13, 9336-9352. [CrossRef] [PubMed]

6. Rohokale, R.; Kshirsagar, U.A. Advanced synthetic strategies for constructing quinazolinone scaffolds. Synthesis 2016, 1253-1268.

7. Badolato, M.; Aiello, F.; Neamati, N. 2,3-Dihydroquinazolin-4(1H)-one as a privileged scaffold in drug design. RSC Adv. 2018, 8, 20894-20921. [CrossRef]

8. Hemalatha, K.; Madhumitha, G. Synthetic strategy with representation on mechanistic pathway for the therapeutic applications of dihydroquinazolinones. Eur. J. Med. Chem. 2016, 123, 596-630. [CrossRef]

9. Li, S.; Ma, J.-A. Core-structure-inspired asymmetric addition reactions: Enantioselective synthesis of dihydrobenzoxazinone- and dihydroquinazolinone-based anti-HIV agents. Chem. Soc. Rev. 2015, 44, 7439-7448. [CrossRef]

10. Vaidya, S.D.; Argade, N.P. Aryne insertion reactions leading to bioactive fused quinazolinones: Diastereoselective total synthesis of Cruciferane. Org. Lett. 2013, 15, 4006-4009. [CrossRef]

11. Jao, C.-W.; Lin, W.-C.; Wu, Y.-T.; Wu, P.-L. Isolation, structure elucidation, and synthesis of cytotoxic tryptanthrin analogues from phaius mishmensis. J. Nat. Prod. 2018, 71, 1275-1279. [CrossRef]

12. Sun, Q.; Xie, L.; Song, J.; Li, X. Evodiamine: A review of its pharmacology, toxicity, pharmacokinetics and preparation researches. J. Ethnopharmacol. 2020, 262, 113164. [CrossRef]

13. Wang, L.; Fang, K.; Cheng, J.; Li, Y.; Huang, Y.; Chen, S.; Dong, G.; Wu, S.; Sheng, C. Scaffold hopping of natural product evodiamine: Discovery of a novel antitumor scaffold with excellent potency against colon cancer. J. Med. Chem. 2020, 63, 696-713. [CrossRef] [PubMed]

14. Zhang, T.; Lai, Z.; Yuan, S.; Huang, Y.-Y.; Dong, G.; Sheng, C.; Ke, H.; Luo, H.-B. Discovery of evodiamine derivatives as highly selective PDE5 inhibitors targeting a unique allosteric pocket. J. Med. Chem. 2020, 63, 9828-9837. [CrossRef] [PubMed]

15. Zhang, X.; Li, Z.; Ding, Q.; Li, X.; Fan, X.; Zhang, G. Alkylamino-directed one-pot reaction of N-alkyl anilines with CO, amines and aldehydes leading to 2,3-dihydroquinazolin-4(1H)-ones. Adv. Synth. Catal. 2019, 361, 976-982. [CrossRef]

16. Cheng, X.; Vellalath, S.; Goddard, R.; List, B. Direct catalytic asymmetric synthesis of cyclic aminals from aldehydes. J. Am. Chem. Soc. 2008, 130, 15786-15787. [CrossRef] [PubMed]

17. Wu, J.; Du, X.-L.; Ma, J.; Zhang, Y.-P.; Shi, Q.-C.; Luo, L.-J.; Song, B.-A.; Yang, S.; Hu, D.-Y. Preparation of 2,3-dihydroquinazolin$4(1 \mathrm{H})$-one derivatives in aqueous media with $\beta$-cyclodextrin- $\mathrm{SO}_{3} \mathrm{H}$ as a recyclable catalyst. Green Chem. 2014, 16, 3210-3217. [CrossRef]

18. Chen, J.-X.; Su, W.-K.; Wu, H.-Y.; Liu, M.-C.; Jin, C. Eco-friendly synthesis of 2,3-dihydroquinazolin-4(1H)-ones in ionic liquids or ionic liquid-water without additional catalyst. Green Chem. 2007, 9, 972-975. [CrossRef]

19. Yoo, C.L.; Fettinger, J.C.; Kurth, M.J. Stannous chloride in alcohol: A one-pot conversion of 2-nitro- $N$-arylbenzamides to 2,3-dihydro-1H-quinazoline-4-ones. J. Org. Chem. 2005, 70, 6941-6943. [CrossRef]

20. Hu, Y.; Wang, M.-M.; Chen, H.; Shi, D.-Q. Efficient and convenient synthesis of spiroindolinone-quinazolines induced by stannous chloride. Tetrahedron 2011, 67, 9342-9346. [CrossRef]

21. Tietze, L.F. Domino reactions in organic synthesis. Chem. Rev. 1996, 96, 115-136. [CrossRef] [PubMed]

22. Nicolaou, K.C.; Montagnon, T.; Snyder, S.A. Tandem reactions, cascade sequences, and biomimetic strategies in total synthesis. Chem. Commun. 2003, 5, 551-564. [CrossRef]

23. Nicolaou, K.C.; Edmonds, D.J.; Bulger, P.G. Cascade reactions in total synthesis. Angew. Chem. Int. Ed. 2006, 45, 7134-7186. [CrossRef] [PubMed]

24. Malacria, M. Selective preparation of complex polycyclic molecules from acyclic precursors via radical mediated or transition metal-catalyzed cascade reactions. Chem. Rev. 1996, 96, 289-306. [CrossRef] [PubMed]

25. Lu, L.-Q.; Chen, J.-R.; Xiao, W.-J. Development of cascade reactions for the concise construction of diverse heterocyclic architectures. Acc. Chem. Res. 2012, 45, 1278-1293. [CrossRef] [PubMed]

26. Wegner, H.A.; Auzias, M. Gold for C-C coupling reactions: A Swiss-Army-knife catalyst? Angew. Chem. Int. Ed. 2011, 50, 8236-8247. [CrossRef] [PubMed]

27. Zi, W.; Toste, F.D. Recent advances in enantioselective gold catalysis. Chem. Soc. Rev. 2016, 45, 4567-4589. [CrossRef]

28. Ohno, H. Gold-catalyzed cascade reactions of alkynes for construction of polycyclic compounds. Isr. J. Chem. 2013, 53, 869-882. [CrossRef]

29. Qian, D.; Zhang, J. Gold-catalyzed cascade reactions for synthesis of carbo- and heterocycles: Selectivity and diversity. Chem. Rec. 2014, 14, 280-302. [CrossRef] [PubMed]

30. Huang, H.; Zhou, Y.; Liu, H. Recent advances in the gold-catalyzed additions to C-C multiple bonds. Beilstein J. Org. Chem. 2011, 7, 897-936. [CrossRef] 
31. Barluenga, J.; Fernández, A.; Satrústegui, A.; Diéguez, A.; Rodríguez, F.; Fañanás, F.J. Tandem Intramolecular HydroalkoxylationHydroarylation Reactions: Synthesis of enantiopure benzofused cyclic ethers from the chiral pool. Chem. Eur. J. 2008, 14, 4153-4156. [CrossRef] [PubMed]

32. Hashmi, S.K.; Bührle, M.; Wölfle, M.; Rudolph, M.; Wieteck, M.; Rominger, F.; Frey, W. Gold catalysis: Tandem reactions of diyne-diols and external nucleophiles as an easy access to tricyclic cage-like structures. Chem. Eur. J. 2010, 16, 9846-9854. [CrossRef] [PubMed]

33. Patil, N.T.; Mutyala, A.K.; Lakshmi, P.G.V.V.; Gajula, B.; Sridhar, B.; Pottireddygari, G.R.; Rao, T.P. Au(I)-catalyzed cascade reaction involving formal double hydroamination of alkynes bearing tethered carboxylic groups: An easy access to fused dihydrobenzimidazoles and tetrahydroquinazolines. J. Org. Chem. 2010, 75, 5963-5975. [CrossRef] [PubMed]

34. Feng, E.; Zhou, Y.; Zhang, D.; Zhang, L.; Sun, H.; Jiang, H.; Liu, H. Gold(I)-catalyzed tandem transformation: A simple approach for the synthesis of pyrrolo/pyrido[2, 1-a][1,3]benzoxazinones and pyrrolo/pyrido[2,1-a]quinazolinones. J. Org. Chem. 2010, 75, 3274-3282. [CrossRef] [PubMed]

35. Lightstone, F.C.; Bruice, T.C. Enthalpy and entropy in ring closure reactions. Bioorg. Chem. 1998, 26, 193-199. [CrossRef]

36. Casadei, M.A.; Galli, C.; Mandolini, L. Ring-closure reactions. 22. Kinetics of cyclization of diethyl ( $\omega$-bromoalkyl) malonates in the range of 4- to 21-membered rings. Role of ring strain. J. Am. Chem. Soc. 1984, 106, 1051-1056. [CrossRef]

37. Galli, C.; Illuminati, G.; Mandolini, L.; Tamborra, P. Ring-closure reactions. 7. Kinetics and activation parameters of lactone formation in the range of 3- to 23-membered rings. J. Am. Chem. Soc. 1977, 99, 2591-2597. [CrossRef]

38. Zhdanko, A.; Maier, M.E. Mechanistic Study of Gold(I)-catalyzed hydroamination of alkynes: Outer or inner sphere mechanism? Angew. Chem. Int. Ed. 2014, 53, 7760-7764. [CrossRef]

39. Kovács, G.; Lledós, A.; Ujaque, G. Hydroamination of alkynes with ammonia: Unforeseen role of the gold(I) catalyst. Angezw. Chem. Int. Ed. 2011, 50, 11147-11151. [CrossRef] 